\title{
MIGRANTES LGBT+ EN LAS CARAVANAS CENTROAMERICANAS HACIA ESTADOS UNIDOS: DILEMAS Y POSIBILIDADES PARA LA CONSTRUCCIÓN DE REDES DE HOSPITALIDAD
}

\section{LGBT+ migrants in the Central American caravans to the United States: dilemmas and possibilities for building hospitality networks}

\author{
Kenia Ortiz Cadena* \\ Nicté Castañeda-Camey ${ }^{* *}$ \\ Rubén García Sánchez ${ }^{* * *}$
}

\begin{abstract}
Resumen. Este artículo tiene como objetivo analizar el escenario de la migración centroamericana en México y en su paso hacia Estados Unidos, con especial atención en el surgimiento del contingente LGBT+ en las caravanas migrantes en 2018. A partir de un estudio cualitativo, se muestran las causas que originan la emigración de personas LGBT+ en Centroamérica, sus experiencias durante el tránsito migratorio, las reacciones generadas en México con su llegada en caravana y las iniciativas de la sociedad para acompañarles. Derivado de esto, se reflexiona sobre algunos elementos asociados a la hospitalidad, para dar cuenta de la complejidad que subyace a este concepto y repensar las formas de construir las micro redes de solidaridad con esta comunidad desde la sociedad civil.
\end{abstract}

Palabras clave: migración centroamericana; caravanas de migrantes $\mathrm{LGBT}+$; redes de hospitalidad y solidaridad.

Abstract. This article aims to analyze the scenario of Central American migration in Mexico and its passage to the United States, with special attention to the emergence of the LGBT+ contingent in migrant caravans in 2018. The causes that originate the emigration of LGBT+ people from Central America, their experiences during the migratory transit, the reactions generated in Mexico with their arrival in caravan and the initiatives of the society to accompany them are revised from a qualitative perspective. Derived from this, it reflects on some elements associated with hospitality,

\footnotetext{
Profesora-investigadora, Universidad de Guadalajara. Jalisco, México. E-mail: keniaortiz@cuc ea.udg.mx. Orcid: https://orcid.org/0000-0002-1159-8939.

** Profesora-investigadora, Universidad de Guadalajara. Jalisco, México. E-mail: ncc863@cucea. udg.mx. Orcid: https://orcid.org/0000-0002-6929-5316.

${ }^{* * *}$ Profesor-investigador, Universidad de Guadalajara. Jalisco, México. E-mail: ruben.garcia. sanchez@academicos.udg.mx. Orcid:. https://orcid.org/0000-0002-0007-3758.
} 
to account for the complexity that underlies this concept and rethink the ways to build micro networks of solidarity and support from civil society.

Keywords: Central American migration; LGBT+ migrant caravans; hospitality and solidarity networks.

\section{Introducción}

Las caravanas de migrantes centroamericanos hacia Estados Unidos (EUA) forman parte del fenómeno de la movilidad de las poblaciones mundiales en el contexto de la fase del desarrollo actual del capitalismo global y las contradicciones entre sus procesos de desterritorialización, que implican un debilitamiento de la soberanía de los estados, con el resurgimiento de propósitos "securitarios" de los estados nación -y del deseo de las masas que los constituyen, para imponer límites a la movilidad de personas-, movilidad que es precisamente resultado de los mecanismos de precarización que imprimen tales procesos de desterritorialización o globalización en primera instancia. A su vez, producto de estructuras sistemáticas que promueven violaciones a los derechos humanos y que impactan diferenciadamente los territorios y los cuerpos (Mesa Transfronteriza Migraciones y Género, 2018).

El fenómeno de las caravanas migrantes centroamericanas no es algo nuevo. Sin embargo, la caravana que salió de San Pedro Sula, Departamento de Cortés, Honduras el 12 de octubre de 2018 con 160 personas y que arribó a Tijuana a finales de noviembre de 2018 con más de 6 mil personas (Colectivo de Observación y Monitoreo de Derechos Humanos en el Sureste Mexicano, 2019; Castañeda, Osorio, Castañeda, 2019; El Colegio de la Frontera Norte, 2018), "se hizo famosa no sólo por su magnitud, sino por el despliegue que generó en los medios de comunicación, en las redes sociales y por las reacciones polarizadas en diversos grupos sociales y políticos (Castañeda, 2016, p. 20), así como "por la campaña construida por Donald Trump para hacerla aparecer como un evento inédito de propósitos aviesos y que representa un peligro para la seguridad estadunidense" (Valenzuela, 2019, p. 40). En esta caravana, Ilamó particularmente la atención la presencia de la comunidad LGBT+, quien se auto identificó públicamente, ocupó un espacio político con identidad propia dentro de este movimiento de inmigrantes y propició el surgimiento de redes de hospitalidad en su trayecto migratorio y a su llegada a EUA.

Desde esta visión, el presente estudio hace un análisis del escenario de la migración centroamericana en México y en su paso hacia Estados Unidos, con especial atención en el surgimiento del contingente LGBT+ en las caravanas migrantes en 2018, se reflexiona sobre el fenómeno a la luz del concepto de hospitalidad, desarrollado por Derrida (2000) a partir de su crítica a Levinas (2006), que la sitúa en una relación indecidible con la hostilidad. 
Nos proponemos mostrar las posibilidades de construcción de redes de hospitalidad desde el nivel micro y de los individuos, en contraste con las políticas migratorias instrumentadas por los estados, que inevitablemente se subsumen a la protección de la soberanía y la seguridad interna. Se valoran las posibilidades para la construcción de tales redes de solidaridad desde una perspectiva ética, que limite o desplace el componente de hostilidad, siempre presente en toda forma de hospitalidad.

El trabajo se divide en tres apartados. En la primera parte se desarrolla una contextualización de la migración centroamericana en México y en su paso hacia EUA, con especial atención al surgimiento de las caravanas organizadas a partir de 2018, se presentan algunos datos de las caravanas más recientes y la situación de inestabilidad de los migrantes varados en Tijuana. En la segunda parte, se describen algunas de las causas que originan la emigración de las personas LGBT+ en Centroamérica, así como sus experiencias durante el tránsito migratorio, las reacciones generadas en México con su llegada en la caravana de 2018 y las iniciativas de la sociedad civil para acompañarlas. En la tercera parte, se analizan los elementos asociados a la hospitalidad para dar cuenta de la complejidad que subyace a este concepto y repensar las formas de construir las micro redes de solidaridad con la comunidad migrante LGBT+ desde la sociedad civil.

Este trabajo es un estudio exploratorio, cualitativo, de carácter descriptivo, respaldado en una epistemología basada tanto en la observación como herramienta para dar solidez a las descripciones de tales movimientos (Aguirre, Jaramillo, 2015), como en el despliegue de diferentes herramientas teórico-analíticas que puedan dar cuenta de los complejos desafíos que se generan para las propias personas en movimiento y las comunidades con las que se relacionan en sus trayectos, así como con las soberanías estatales que intervienen inevitablemente en todo proceso de movilidad. En sintonía con ello, primero se buscó recabar una serie de datos empíricos asociados a los procesos de movilidad y las consiguientes formas de hospitalidad que experimentan los migrantes LGBT + . Para ello se realizó un trabajo de observación en el Albergue Temporal "Casa Arcoíris" en Tijuana y una entrevista con un informante clave en abril de 2019. También se obtuvieron testimonios informales y material audiovisual sobre "Casa Migrante Respetttrans" de Ciudad Juárez, por parte de su fundadora, durante el primer semestre de 2020. Por otra parte, se recuperaron las experiencias de los migrantes LGBT + a través del foro online "Central American Caravans: Inside LGBTQ Testimonies of Contention, Survival and Resilience", organizado por el colectivo "Diversidad sin Fronteras" el 28 de mayo de 2020. Esto con la finalidad de identificar las circunstancias que motivaron su migración, sus vivencias durante el trayecto migratorio con la caravana y las acciones de hospitalidad y solidaridad que recibieron por parte 
de otros migrantes y asociaciones civiles. Para verificar las reacciones generadas entre la población con la llegada de los migrantes LGBT+ a Tijuana en 2018, se llevó a cabo una revisión y análisis en YouTube y Facebook; se recuperaron los comentarios señalados sobre migrantes, identificando las connotaciones xenófobas, racistas, homófobas, entre otras ${ }^{1}$.

Simultáneamente, se desarrolló una contextualización de la migración centroamericana en México y en su paso hacia EUA, con especial atención a los antecedentes, el surgimiento de las caravanas migrantes, la participación reciente de los migrantes LGBT + en éstas y las iniciativas de la sociedad civil para crear redes de apoyo y solidaridad hacia esos migrantes. Para llevar a cabo este proceso, se hizo una indagación en fuentes documentales y electrónicas, páginas web, periódicos y revistas.

Una vez recabada y analizada la información empírica y documental, se realizó una fundamentación teórica del concepto de hospitalidad, concebido como una de las bases para el mantenimiento de los procesos de movilidad corporal y subjetivante en que se ven envueltos los integrantes de la comunidad LGBT+ migrante. Mediante ello, se busca vislumbrar algunas de las posibilidades para la construcción de redes de hospitalidad a nivel micro y desde las propias comunidades de tránsito, destino y retorno, dada la imposibilidad de que ello ocurra en el nivel de los estados-nación.

\section{Migración centroamericana en México y en su tránsito hacia Estados Unidos}

En Latinoamérica las fronteras se han configurado como espacios regionales caracterizados por elementos históricos, demográficos, económicos y sociopolíticos. La migración internacional, intrarregional y entre los países limítrofes latinoamericanos es un fenómeno de antaño. En las últimas décadas se presentan nuevos escenarios, modalidades y patrones migratorios. Se puede decir que la migración latinoamericana, se ha intensificado y diversificado, se presenta circular, transfronteriza, de retorno, indocumentada, con una diversidad de actores (Canales, 2013; Herrera, Sørensen, 2017; Yepez, 2014), sujetos sociales y comunidades que se han incorporado y hecho visibles.

Asimismo, en estas fronteras latinoamericanas se han reconfigurado espacios y procesos orientados a regular, vigilar y a condicionar la movilidad. Todo esto bajo la lógica de control "securitario" en la que se articulan situaciones como la admisión, el asilo, el refugio, la espera, la denegación de

La revisión se realizó en octubre de 2019 en redes sociales de diversos medios de comunicación donde se había informado sobre la presencia del colectivo de migrantes LGBT+ en las caravanas, entre los cuales la revista VICE (para mayor información ver < https://www.youtube. $\mathrm{com} /$ watch? $=\mathrm{g} 68 \mathrm{XyW}$ wLqf8 $>$ ). 
visado, la deportación, la detención, la expulsión o el hostigamiento (Brandariz, Mendiola, 2019).

Específicamente la migración centroamericana en México y en su tránsito hacia EUA, se ha definido históricamente por sus numerosos flujos migratorios interregionales. En su origen (segunda mitad del siglo XIX), se destacaba por ser temporal, agrícola y no masiva (Casillas, 1991). Las familias que provenían de comunidades indígenas se desplazaban de Guatemala a la frontera sur de México para trabajar en el cultivo de caña de azúcar, café y algodón. Este tipo de migración prevaleció hacia la década de los setenta del siglo XX, y gradualmente debido a las guerras civiles en Guatemala, El Salvador y Nicaragua, se fueron produciendo desplazamientos forzosos en toda la región. Convirtiéndose en una fuga masiva a México y en tránsito hacia EUA (Carrasco, 2013; Morales, 2013).

Una vez terminados los conflictos armados, en 1992 para El Salvador, y en 1996 para Guatemala, se esperaba que los desplazados tuvieran un retorno migratorio permanente y voluntario. Sin embargo, estos retornos fueron paulatinos, individuales y forzados (deportación o remoción desde EUA). Ya en sus lugares de origen, las personas no encontraron una política clara de reinserción e incorporación a la sociedad (Martínez, Cobo, Narváez, 2015).

Al mismo tiempo, en la región se produce un clima de violencia generalizada provocada por los miembros de las pandillas, que igualmente fueron deportados de EUA en los años anteriores. Aunado a esto, se suman los desastres naturales en 1998 (Huracán Mitch), lo cual desató una nueva corriente migratoria, que reactivó el circuito migratorio internacional de tránsito no documentado por México hacia EUA (Carrasco 2013; El Colegio de la Frontera Norte, 2018; Narváez, 2007).

A partir del año 2000, se puede hablar del inicio de una nueva etapa para la migración centroamericana de tránsito en México hacia EUA, la cual se podría denominar "heterogénea" (Martínez, Cobo, Narváez, 2015), caracterizada por:

a) Las diversas acciones emprendidas a partir del 09/11 en 2001 marcaron una era para las políticas migratorias globales, ubicándose EUA en los ejes de "migración y seguridad nacional" y de criminalización de la migración indocumentada. Se da una transformación orgánica en los aparatos institucionales migratorios: desaparece el Immigration and Naturalization Service (INS) y se funda el Department of Homeland Security (DHS) que concentra al Immigration and Customs Enforcement (ICE), el Customs and Border Protection (CBP) y el U.S. Citizenship and Immigration Services (USCIS). Esta política de securitización y control de las fronteras propició una migración con mayores costos económicos y con más vínculos entre el crimen organizado y el migrante (Narváez, 2019, p. 15). 
b) Los estragos que causó el Huracán Stan (2005) en la región centroamericana y en algunos estados del sur México, en los que se vio afectada gran parte de las vías del tren en Tapachula, trajo consigo un desvío de la "ruta tradicional de paso por Chiapas" hacia otras conexiones ferroviarias en Tabasco, lo cual significó un aumento en la inseguridad del trayecto migratorio.

c) La industria del crimen organizado que ha ejercido desde 2008 el control absoluto de las rutas migratorias (Chiapas, Tabasco, Veracruz y Tamaulipas). Esto ha encarecido los costos del traslado, el cruce de la frontera y ha incluido el secuestro masivo y extorsión de los migrantes centroamericanos (Martínez, Cobo, Narváez, 2015).

d) El decreto de la Ley de Migración en México en 2010 y la publicación en 2012 del Reglamento de Ley de Migración y la fundación de la Unidad de Política Migratoria de la Secretaría de Gobernación, lo que repercute en cambios en la estructura burocrática y en el diseño de políticas.

e) La puesta en marcha en México del Programa Frontera Sur en 2014, producto de la crisis política de los "menores no acompañados", el cual se fundamenta en una visión de seguridad pública y nacional enfocada en criminalizar la migración centroamericana indocumentada. Los migrantes centroamericanos son detenidos y deportados sin considerar su vulnerabilidad (Castañeda, 2016; Mecanismo Permanente de Monitoreo de la Frontera Sur México-Guatemala, 2018; Narváez, 2019).

f) El anuncio de EUA el 20 de diciembre de 2018 del protocolo "Quédate en México" (también conocido como Migration Protection Protocols MPP y Remain in Mexico), y la respuesta inmediata de México que indica que "por razones humanitarias" el gobierno mexicano lo autorizaba ${ }^{2}$. Con lo cual se obliga a regresar a México a miles de migrantes, en situaciones de vulnerabilidad, en lo que se procesan en EUA sus solicitudes de asilo (Instituto para las Mujeres en la Migración, 2020).

\section{La conformación de las caravanas migrantes centroamericanas}

Como se mencionó anteriormente la migración centroamericana en grupo no es nueva, siempre ha existido, pero los migrantes eran menos visibles y pasaban aparentemente "desapercibidos". Esa invisibilidad desencadenó todo tipo de abusos por parte de las autoridades mexicanas y del crimen organizado; por ello tomaron la decisión de hacerse notar y hasta de convocar a medios de comunicación para que documentaran su trayecto (Serrano, 2019).

Esta lucha migrante en forma de "caravana" tiene como antecedente la "Caravana de las Madres Centroamericanas", que desde 1999 se han organizado

2 Este protocolo se implementó formalmente el 25 de enero de 2019. 
ininterrumpidamente para buscar a sus hijos e hijas desaparecidos(as) en México. Y concretamente tiene como precedente la conformación del autodenominado "Viacrucis Migrante", que de 2011 hasta 2015³, se constituyó como una forma novedosa de lucha que usa la "performatividad del viacrucis religioso" para vincularlo con lo político y hacer visible algunas demandas y estrategias colectivas concretas: exigir que se garanticen los derechos humanos de las personas migrantes y su tránsito libre por territorio mexicano; así como denunciar las violaciones a los derechos humanos y los delitos que se cometen en México (Varela, McLean, 2019, p. 175; Vargas, 2016).

Los Viacrucis se organizaban en México por parte de activistas católicos y otras asociaciones, entre ellos "Pueblos sin Fronteras". En estos se recreaban todos los aspectos del drama humano concentrado en los migrantes: la deportación, las travesías por el desierto, las violaciones sexuales a mujeres, la trata de blancas, las desapariciones, el abuso y explotación por parte de los patrones que se aprovechaban de su condición migratoria irregular, entre otros (Valenzuela, 2019).

Sin embargo, a partir de 2014 los autodenominados caravaneros empiezan a organizar una nueva forma que rebasa al viacrucis: "las caravanas de migrantes". Se genera un cambio de estrategia entre la logística performativa del viacrucis y la que los migrantes idean. Es decir, se produce una nueva forma de lucha migrante de "autodefensa" para llegar a EUA sin polleros $y$, en la medida de lo posible, sin cuotas de sufrimiento social y, sobre todo, con demandas concretas: "respeto al derecho al asilo, al refugio y la libertad de circulación y de exigir con sus cuerpos el derecho a preservar su vida y que esta se habite con dignidad" (Varela, McLean, 2019, p. 180-182).

Dentro de la conformación de estas caravanas se destaca la que empezó a convocarse el 5 de octubre de 2018 en Honduras por medio del despliegue generado en los medios de comunicación, en las redes sociales y por las reacciones polarizadas en diversos grupos sociales y políticos (principalmente Facebook y WhatsApp), denominada: "Marcha del migrante"; "No nos vamos porque queremos: nos expulsa la violencia y la pobreza". Lo novedoso no fue la forma de migrar en caravana, sino fue que, por iniciativa de los propios desplazados centroamericanos, la marcha se gestó en los territorios de donde son originarios, en este caso en Honduras (Castañeda, Osorio, Castañeda, 2019; Castañeda, 2016).

Esta caravana salió de San Pedro Sula, Departamento de Cortés, Honduras el 12 de octubre de 2018 con 160 personas y arribó a Tijuana a mediados de

3 De acuerdo con Vargas (2016) algunos de los promotores de los viacrucis migrantes fueron objeto de amenazas directas por parte del crimen organizado y de agentes estatales y dejaron de realizarlos en 2015. 
noviembre de 2018 con más de 6 mil personas. Al inicio todos sus integrantes caminaban juntos, pero fue a partir de la entrada a la Ciudad de México que empezaron a dispersarse y a viajar en grupos de decenas y centenares de personas. Se ha documentado en muchos medios, que en la entrada a México de la primera caravana fue el 19 de octubre de 2018 y la llegada de sus primeros integrantes a Tijuana el 10 de noviembre de 2018. La movilidad por México se dio principalmente a través de transportes públicos, de "aventones", en transporte de carga, en autobuses proporcionados por organizaciones sociales o por gobiernos estatales y a pie. Se destaca aquí también una emergencia tanto de ejercicios de hospitalidad por parte de la ciudadanía, como muestras de racismo y xenofobia (El Colef, 2018)

A Tijuana llegó primero un grupo de poco más de 80 personas identificadas como de la comunidad LGBT + . Posterior a esto, fueron llegando grupos de cientos de personas. El 14 de noviembre el gobierno de Tijuana abrió un albergue temporal en la Unidad Deportiva Benito Juárez, a cargo de la directora del Sistema Nacional para el Desarrollo Integral de la Familia (DIF), quien anunció que tenía cupo para 2000 personas. A medida que llegaban los migrantes de la caravana a Tijuana eran ubicados en esta unidad deportiva, alcanzando hasta 6,151 personas el día 28 de noviembre (EL Colef, 2018).

Se puede calcular que llegaron tres "oleadas" más de caravanas a partir de 2018. Sin embargo, debido a la cambiante política migratoria del gobierno mexicano, se han presentado complicaciones en su tránsito y/o estadía por México. Con cada caravana las autoridades han implementado distintas estrategias para brindar o eliminar mecanismos de protección humanitaria de forma casi aleatoria o arbitraria. La primera caravana de 2019 se benefició de una estrategia que pretendió ser más "ordenada y humanitaria" al regularizar a las personas y otorgarles una "visa humanitaria con validez de un año"; pero a partir de marzo, a las siguientes caravanas, se les negó este procedimiento (Fernández De La Reguera, 2019).

Todo este escenario de endurecimiento de las políticas migratorias en ambos países durante los últimos tres años se ha tornado aún más complicado con el auge de la COVID-19. Por ejemplo, el 19 de marzo de 2020 se cerró la frontera entre Estados Unidos y México en ambas direcciones para todos los viajes "no esenciales" como el turismo. Posterior a esto, el 20 de marzo se inició en EUA un nuevo protocolo para deportaciones aceleradas a México de migrantes mexicanos, guatemaltecos, salvadoreños y hondureños, incluyendo a los menores de edad no acompañados y solicitantes de asilo, aprehendidos por agentes de la patrulla en la zona fronteriza (Miroff, 2020). El 24 de marzo,

4 Para mayor precisión en la cronología de esta caravana revisar: <https://observatoriocolef.org/ infograficos/cronologia-de-la-caravana-centroamericana/>. 
se suspendieron hasta nuevo aviso todas las audiencias de asilo del programa Protocolos de Protección al Migrante. Todas estas medidas han provocado una dinámica de gran retención de migrantes con diferentes perfiles varados en Tijuana, lo cual implica mayores riesgos de violentar sus derechos humanos desde ambos lados de la línea fronteriza (Del Monte, Mckee, 2020).

\section{Migración de personas LGBT+ centroamericanas hacia Estados Unidos}

La discriminación por orientación sexual es una práctica muy difundida en Centroamérica. De acuerdo al Centro por la Justicia y el Derecho Internacional (2013), entre las múltiples causas que han permitido y propiciado el panorama de violencia contra las personas LGBT+ en Costa Rica, Honduras y Nicaragua, están la existencia de una sociedad excluyente de la diversidad sexual, la falta de sensibilidad y preparación sobre la situación de esta población por parte de los servidores públicos, y la presencia de vacíos legales para garantizar sus derechos. Esta circunstancia se presenta también en El Salvador, donde según indica Gómez (2017), prevalece el silencio por parte del gobierno sobre los homicidios a personas LGBT+.

Por otra parte, las pandillas de la Mara Salvatrucha y Barrio 18 ejercen sistemáticamente violencia hacia las personas LGBT+ en Centroamérica. Gómez (2017) identifica cuatro criterios bajo los que estos grupos delictivos llevan a cabo las acciones de violencia contra personas salvadoreñas LGBT+: 1) el homicidio como ritual de iniciación para ser aceptado en una pandilla; 2) la subsecuente impunidad que impera con respecto a los crímenes perpetrados hacia estas minorías sexuales; 3) el pleno rechazo a los gays o travestis; y 4) la extorsión bajo amenaza de muerte ejercida contra estas personas, a quienes se les suele cobrar dinero por sus negocios.

Lo anterior ha hecho que miles de personas LGBT + se vean obligadas a salir de su país para buscar asilo en EUA, país que ha reconocido desde hace más de dos décadas la persecución por orientación sexual como motivo para otorgar el asilo político. En el año 2011, según señala Portman y Weyl (2013), entraron 81,372 refugiados y solicitantes de asilo a EUA, de los cuales tres mil podrían identificarse como LGBT+, ya que muy pocos de ellos revelan su orientación sexual o identidad de género, por temor a ser discriminados ${ }^{5}$.

\footnotetext{
5 De acuerdo a las políticas del gobierno de Estados Unidos, las figuras de asilo político y refugio aplican para las personas que han sido o pueden ser perseguidas por pertenecer al colectivo LGBT+. El asilo puede solicitarse en cualquier puerto de entrada al país (aéreo, terrestre o marítimo), ante un oficial de inmigración o estando dentro de Estados Unidos, mientras que para requerir el refugio es obligatorio estar fuera de Estados Unidos y el propio país. Y como se indicó en el primer apartado de este artículo, con la entrada en vigor del protocolo "Quédate en México" en 2019, se obliga a los solicitantes de asilo a permanecer en México mientras se procesa su trámite.
} 
No obstante, con la reciente incursión de las caravanas centroamericanas de migrantes que buscan ingresar a EUA, observamos un cambio en este patrón, puesto que los migrantes LGBT+ se han organizado como colectivo. En la caravana que salió el 12 de octubre de 2018 de Honduras y que arribó a Tijuana en noviembre del mismo año, llamó particularmente la atención la presencia de la comunidad LGBT+, quien se auto identificó públicamente y ocupó un espacio político con identidad propia dentro de este movimiento de migrantes: "el grupo venía muy empoderado, organizado por sus líderes, y en conjunto decidían cómo iban a hacer su trayecto. Este grupo no se había organizado desde su salida, en realidad se fue conformando en su paso hacia Estados Unidos" (J.L. Villa, comunicación personal, 24 de abril, 2019).

\section{Xenofobia, discriminación y violencia hacia los migrantes LGBT+ en su paso por México}

Tanto dentro de las caravanas como entre la población mexicana, los migrantes LGBT + han sufrido discriminación y violencia. Muestra de ello son las actitudes de discriminación que recibieron un grupo de más de 80 migrantes en la caravana en el 2018, quienes debido a ello decidieron separarse de este colectivo en la ciudad de México (The Objective, 2018). Al llegar a un albergue en Tijuana, y sufrir nuevamente discriminaciones por parte de la caravana, decidieron rentar una casa en una zona habitacional de "Playas de Tijuana". El grupo recibió amenazas e intimidaciones por parte de los vecinos, quienes no aceptaron su presencia en el barrio, ya que consideraban que los migrantes LGBT+ representaban un peligro para su seguridad (La voz de la frontera, 2018).

Además, con la presencia del grupo LGBT+ en las caravanas, han surgido miles de comentarios racistas, xenófobos y homófobos que han circulado en las redes sociales como YouTube y Facebook, donde se señala a estas personas como: "violadores", "traficantes", "pedófilos", "una amenaza para el país", "jotos a los que nadie quiere en México", "basura", "depravados", "lacras", "porquería", "pervertidos sexuales", como un "cáncer" para la sociedad, "sidosos", entre otros. Este sector de la población mexicana tiene una opinión negativa de las caravanas, y además discriminan cuádruple a los migrantes LGBT+: por su condición migrante, por su lugar de nacimiento, por su orientación sexual y por su clase social.

En este contexto hostil hacia los migrantes LGBT+ en México, y considerando el clima de violencia generalizado debido a la presencia de narcos y grupos delictivos, las agresiones a estas personas se han potenciado. Según muestra Mastrogiovanni (2014), la mayoría de estos migrantes son incluso secuestrados y obligados a prostituirse o colaborar con el crimen organizado. 


\section{La organización colectiva entre migrantes LGBT+}

Es importante señalar, que la organización colectiva de los migrantes LGBT + ha permitido contrarrestar los efectos de la discriminación y violencia que encuentran desde la salida de su país hasta la llegada a EUA, creándose así un espacio de hospitalidad y acompañamiento. Los migrantes LGBT+ suelen salir solos de su país y en las caravanas han identificado un espacio para unirse y sentirse más seguros, una colectividad que representa "esperanza", "sumar entre las personas", "sueños", "superaciones de todo tipo de personas", "unión", "amor" y "desafío" (Foro online "Central American Caravans: Inside LGBTQ Testimonies of Contention, Survival and Resilience", 28 de mayo, 2020). Para Rosa la caravana ha simbolizado "valor, fuerza y unión," ya que según relata:

Es una gran masa de personas las que vienen ahí en la caravana, que sentimos que si venimos solas no vamos a lograr llegar hasta Estados Unidos, porque en el camino solas somos muy vulnerables. En caravana uno siente (...) unión, esperanza, porque por ejemplo, yo toda mi vida tuve la esperanza de venirme a este país, pero no lo hice por el miedo a ser asesinada, a ser violada, a seguir siendo discriminada en el camino. Al darme cuenta que venía en la caravana lo que sentí fue fuerza, me sentí más protegida porque son tantas personas que entre todos se cuida uno, y pues en la caravana venimos de todo. (Foro online "Central American Caravans: Inside LGBTQ Testimonies of Contention, Survival and Resilience", 28 de mayo, 2020)

Las personas LGBT + que se unieron a las caravanas, en la mayoría de los casos han formado grupos internos para protegerse entre ellas mismas de la discriminación experimentada dentro de la caravana y fuera. Sin embargo, los procesos para crear colectividad pueden variar en función de las personas, las circunstancias y el lugar donde se encuentran; en algunos momentos estos procesos pueden motivar la creación de colectivos de diez migrantes LGBT+, pero en otros sólo de dos. Y es importante considerar que, aunque cada vez son más los migrantes que se identifican como LGBT+, lo cual está permitiendo que se generen estas agrupaciones, todavía muchos temen a ser reconocidos como tales y ser discriminados, por lo que mantienen anónima su orientación sexual e identidad de género.

\section{Iniciativas de la sociedad civil para acompañar a los migrantes LGBT+}

También el surgimiento de asociaciones en México y Estados Unidos ha permitido contrarrestar los efectos de la discriminación y violencia experimentada por los migrantes LGBT+. Han nacido nuevas iniciativas de apoyo para estos migrantes, como los albergues "Casa Arcoíris" en Tijuana en 2017 y "Casa Migrante Respetttrans" en Ciudad Juárez en 2018. En el primer sitio se reciben a personas de la comunidad LGBT + que tienen la intención de ingresar a EUA o permanecer en México por asilo, a quienes acaban de llegar a 
Tijuana para establecerse en esta ciudad o aquellos que fueron deportados del país vecino. La mayoría de los migrantes que están en el albergue han iniciado su trámite para requerir asilo en EUA y provienen de El Salvador, Guatemala y Honduras. El albergue está integrado por diversos colectivos LGBT + y vinculado con otros, uno de ellos es "Enclave caracol", que orienta a las personas para iniciar su proceso de asilo (J.L. Villa, comunicación personal, 24 de abril, 2019).

"Casa Migrante Respetttrans" es un espacio para dar refugio a migrantes transgénero, transexuales, mujeres lesbianas y sus hijas e hijos. Además, el personal del albergue asesora a estas personas en su proceso migratorio, en colaboración con asociaciones civiles en EUA que dan seguimiento a casos de asilo y deportaciones. Respetttrans es parte de una red de asociaciones que exigen al ICE y a las autoridades migratorias de México, implementar políticas contra la detención de personas transgénero, ya que se ha registrado la muerte de tres mujeres trans bajo custodia de las autoridades migratorias: una en Tapachula, México y dos en Cibola, EUA. Y de acuerdo a un estudio de la Oficina de Responsabilidad Gubernamental de este último país, en el 2013 los migrantes trans detenidos representaban uno de cada cinco asaltos sexuales confirmados bajo custodia del ICE (Love, 2019).

Por otra parte, han surgido colectivos como "Diversidad sin Fronteras", que hacen un acompañamiento a través de las redes sociales para estos migrantes, manteniéndolos al tanto de los posibles puntos de peligro en su tránsito a EUA, brindando asesoría legal para organizar los expedientes de asilo y recabando dinero para llevar utensilios de aseo personal a los migrantes LGBT+ que están en los centros de detención del ICE. Asimismo, hay organizaciones en EUA, como "Al otro lado", que está integrada por abogadas y abogados que están dando seguimiento a los casos de los migrantes centroamericanos, y que colaboran con la comunidad LGBT+. También, la organización "Raíces" brinda acompañamiento a migrantes LGBT+ en su proceso de asilo. De hecho, hay una colaboración importante entre el albergue "Casa Arcoíris", "Casa Migrante Respetttrans" y estas asociaciones de EUA; para en conjunto dar soporte a los migrantes LGBT+ que están en los centros de detención, esperando que se resuelva su solicitud de asilo.

Estas asociaciones tienen un papel importante en la hospitalidad hacia migrantes LGBT+. "Casa Arcoíris" y "Casa Migrante Respetttrans" brindan un entorno seguro para estos migrantes que han sufrido discriminación por parte de un sector de la sociedad en México y en las mismas caravanas. Dado que todavía hay migrantes LGBT+ que mantienen en anonimato su orientación sexual y/o su identidad de género, el trabajo en estos albergues es relevante, ya que es un espacio para que estas personas puedan expresarse sin limitaciones, lo cual les brinda mayor seguridad para enfrentar su proceso por asilo (J.L. Villa, comunicación personal, 24 de abril, 2019). Por otra parte, la asesoría legal es 
central en el proceso de asilo, puesto que se estima que el 90 por ciento de las solicitudes se pierden cuando no están representadas por una abogada u abogado.

Muchas de estas asociaciones en EUA, forman parte de redes de apoyo para migrantes, como "Santa Fe Dreamers" que ofrece servicios legales. Este colectivo tiene un programa especial de representación legal y defensa colaborativa para migrantes LGBT + detenidos en todo EUA a través de una red de asociaciones locales. Además, ha impulsado un proyecto de hospitalidad hacia los migrantes, que consiste en obtener el apoyo de "patrocinadores", que son personas en EUA que aceptan acoger en sus hogares a un solicitante de asilo que no cuente con una red familiar o social en este país (Santa Fe Dreamers, s.f.).

Algunos testimonios de personas que han emigrado de sus países para obtener asilo político en EUA, son muestra de todo lo antes descrito. Gio es de El Salvador, salió de su país por discriminación y por amenazas de pandillas puesto que no quería vender drogas. Gio considera que todos los migrantes LGBT han experimentado discriminación y violencia en su trayecto migratorio, sin embargo, hablan poco del trato que reciben en los centros de detención en Estados Unidos. Según indica Gio:

Estar dentro de las manos del ICE es muy difícil, es una experiencia inimaginable, pues cuando yo entré con el grupo de todas las chicas trans, fue una experiencia la verdad muy fea, porque la pasamos de detención en detención. Estuvimos en El Paso casi un día sin comer (....). Ya estando en Cibola, por la fecha de diciembre que es una etapa muy fría por la nieve y todo lo demás, recuerdo que en el centro de detención no ponían la calefacción, eran días que nosotros dormíamos con aquel frío insoportable. (Foro online "Central American Caravans: Inside LGBTQ Testimonies of Contention, Survival and Resilience", 28 de mayo, 2020)

Actualmente Gio está haciendo los trámites para obtener asilo político. Debido a la intervención de "Diversidad sin Fronteras" y otros colectivos él obtuvo el apoyo de un patrocinar en Massachusetts, donde ahora vive, señala: "después de una situación triste y tormentosa puedo decir que estoy bien, que estoy tranquilo, en un lugar seguro que no me pueda pasar nada" (Foro online "Central American Caravans: Inside LGBTQ Testimonies of Contention, Survival and Resilience", 28 de mayo, 2020).

Chantal es de México, y dada la discriminación que sufría entre su familia, decidió migrar a Tijuana, donde enfrentó malos tratos por parte de la policía, quien "la golpeaba" y "la querían desaparecer". Chantal estuvo un año y cuatro meses en los centros de detención, le negaron el asilo; pero gracias al apoyo de "Diversidad sin Fronteras" utilizó el recurso de apelación. Chantal considera que el apoyo de los colectivos en Tijuana y Agua Prieta le permitió luchar y mantenerse con vida, darse cuenta que "no estaba sola", "que había alguien a su lado", esto la hacía "fuerte adentro" (Foro online 
"Central American Caravans: Inside LGBTQ Testimonies of Contention, Survival and Resilience", 28 de mayo, 2020).

\section{Construcción de redes de hospitalidad para migrantes LGBT+}

En un contexto de rechazo y criminalización de la migración y los cuerpos que la encarnan cabe preguntarse icómo es posible construir en un nivel normativo las redes políticas de solidaridad con los otros desde un contexto dominado por el pensamiento político occidental que concibe la responsabilidad solo al seno del núcleo familiar, el clan o la nación? Rosine Kelz plantea que la creación de redes de solidaridad entre ciudadanos con no-ciudadanos parte de la noción de responsabilidad por los "otros" como incentivo para la creación de formas de asociación política más abiertas. Esto entra en contradicción directa con el concepto de soberanía, central en el pensamiento moderno sobre el individuo y el Estado (Kelz, 2015, 2016). El problema ético fundamental es cómo extender los lazos de solidaridad hacia los no miembros dentro de comunidades políticamente establecidas.

Kelz (2015) plantea, en contraste con la concepción tradicional del sujeto soberano -que se supone actúa y piensa de manera autónoma-, que el sujeto no soberano se define como tal por su relación con el otro, para Levinas (2006) el mismo entendimiento del mundo está supeditado a esa relación. Ello implica que el sujeto sólo puede constituirse en conexión con otro sujeto y no puede encontrar sentido únicamente en su propio ser-en-el-mundo, sino que es sólo mediante la percepción de un otro trascendente que el hombre puede comprender su existencia en el tiempo y el espacio. Entonces dado que el otro está antes del sujeto y este solo puede establecer su ser en relación, existe una deuda hacia el otro, que es tanto una imposición al sujeto como la posibilidad de su agencia moral y su subjetividad. Para Levinas, en el encuentro entre personas la cara del otro es la que expresa tal demanda ética. La cara revela un universal, una alteridad y vulnerabilidad infinitas que se acompañan del mandato de no matar al otro. El espacio ético se produce por el encuentro cara a cara que instituye la relación entre la infinitud y la alteridad (Casey, 2006).

Kelz (2015) plantea que Butler llega a una conclusión similar desde la narrativa de la precariedad y la dependencia, al afirmar que el sujeto es solo por la vía de su inevitable relación con los otros, los que también le otorgan el reconocimiento de ser un portador de derechos. La relación con los otros desafía permanentemente las fronteras externas del ser. La precariedad (precariousness) para Butler (2004) es una dimensión socio-ontológica de todos los cuerpos, lo que pone de relieve es que como seres relacionales, vulnerables y finitos dependemos de los otros en múltiples formas, sin embargo, 
la precariedad no nos hace a todos iguales, la forma en que los individuos se exponen a la precariedad difiere en la contingencia de los cuerpos (precarity).

Derrida (2000) plantea que la hospitalidad absoluta de Levinas implica ofrecer algo al otro sin tomar en cuenta sus particularidades de género o extranjería, así se llegue con o sin invitación (Madura, 2016). Pero para pasar de esta concepción ética ideal a la realidad es necesario tomar las particularidades en cuenta. El dar asilo, alimentos o derechos de ciudadanía requiere limitar a ciertos individuos mientras se incluye a otros, el dilema ético implica decidir a quién se le da y a quien no. La hospitalidad absoluta es un imperativo ético a la vez que una imposibilidad política. Para Derrida la hospitalidad absoluta solo puede ser poética, si solo somos hospitalarios con unos se viola la ley de la hospitalidad absoluta, pero si intentamos ser hospitalario con todos no podemos serlo con ninguno. Derrida (2000) no busca rechazar el concepto de hospitalidad absoluta sino preservarlo como la meta ideal de nuestras relaciones intersubjetivas, pero tomando en cuenta que cualquier fórmula para lograr la hospitalidad implica una violación de la misma.

Para Kelz, la hospitalidad de Derrida pone en duda la posibilidad misma de estados nación soberanos y justos. La relación del yo con el otro se vincula directamente con el sujeto no soberano y la ética de la migración (Derrida, Dufourmantelle, 2000). Para Derrida el sí mismo es al ocupar simultáneamente el sitio del otro dándole la bienvenida a su espacio. En esta simultaneidad se traspasan las fronteras haciendo que el uno y el otro se establezcan en su no-soberanía como relacionalmente dependientes. El uno no comienza desde una identidad sustancial que constituya la base de su capacidad de dar la bienvenida, sino que es la hospitalidad en sí misma la que le define y constituye como sujeto, el sujeto se convierte así en la apertura al otro. Kelz plantea que podemos objetar que sólo puede darse la bienvenida al otro desde un espacio que presupone un sujeto soberano, es a partir de este hecho que Derrida pone límites a su idea de la hospitalidad incondicional y advierte de una situación en que el anfitrión pierde el sentido de ser el amo de su propia casa y que puede volver hostil la relación entre el huésped y el anfitrión. De ese modo, cualquiera que atente contra mi soberanía como anfitrión se vuelve un extranjero indeseable. Para evitar eso Derrida menciona que el anfitrión establece condiciones o leyes de hospitalidad, pero esto introduce una tensión irresoluble entre esas las leyes que regulan la hospitalidad y la hospitalidad incondicional, lo que hace que esta última se vuelva imposible, dado que su condicionamiento incluso puede distorsionar o pervertir la hospitalidad (Kelz, 2015). Esto es exactamente lo que ocurre con los estados europeos y otros más que buscan cumplir con las obligaciones humanitarias 
hacia los refugiados al mismo tiempo que limitan su acceso a sus espacios nacionales soberanos "securitarios".

Desde esta perspectiva basada en Levinas, Butler y Derrida es que Kelz construye la posibilidad de un argumento moral mediante el que los ciudadanos de países occidentales desarrollados deberían interrogar de manera crítica su derecho a la exclusividad en un territorio; además, el entendimiento del otro como un elemento constitutivo del ser, permite plantear la responsabilidad de manera independiente del conocimiento que se tenga o no sobre la otra persona, lo que importa es comprender que el otro es vulnerable. Estos elementos permiten cuestionar los fundamentos de la soberanía del estadonación y realizar la crítica de las prácticas políticas de control fronterizo.

La hospitalidad es ya un concepto central para pensar el paradigma emergente de la movilidad (Gibson, 2016) desde el cual se han de imaginar las respuestas éticas para determinar los modos de recibir al extranjero y distinguir, como lo hace Derrida, entre ética como hospitalidad y las leyes o políticas de la hospitalidad. Derrida incluso plantea el término hostipitalidad (Bida, 2018; Derrida, 2000) a partir de la deconstrucción de los opuestos binarios de hostilidad y hospitalidad (ambos términos tienen un origen común en hostis como anfitrión y hostis como enemigo) y plantea que la hospitalidad es la base misma de la cultura y no sólo una ética frente a los otros, la ética es coextensiva a la hospitalidad. La hospitalidad mantiene un velo de desigualdad, especialmente cuando se basa simplemente en la amabilidad y generosidad del anfitrión, a modo de caridad. La importancia del término hostipitalidad es que no oculta la violencia que encarna cualquier hospitalidad que no sea incondicional, y que está latente en toda forma de bienvenida que plantea los límites al huésped de no convertir la casa que le acoge en su casa.

Si el concepto de hospitalidad incondicional implica la apertura al otro sin límites, entonces se debe pensar el concepto de hospitalidad (Still, 2013) desde el punto de vista de la diversidad étnica, lo femenino y el género no binario (Michaud, 2018), lo alter y lo queer (Blevins, 2009). Es fundamental reconocer que la mayoría de políticas, servicios y previsiones dirigidos a los migrantes asumen que estos son heterosexuales y comenzar a explorar las múltiples conjunciones entre la sexualidad y la migración (Luibhéid, 2008).

La hospitalidad hacia los migrantes LGBT+ implica la construcción de una ética de la diferencia en todos los sentidos y niveles de la sociedad y contrarrestar el discurso de odio hacia todos los grupos LGBT+, no sólo los migrantes. Para Deleuze y Guattari (1997) ello solo puede darse desde una perspectiva molecular, no puede existir una solución desde el Estado, debemos convertirnos en máquinas de guerra nómadas y librar esa batalla en cada ámbito y resquicio de la sociedad, la hospitalidad debe estar diseminada 
en cada uno. Para Deleuze (2005) "Il]a ley del Estado no es la del Todo o Nada (sociedades con Estado o sociedades contra Estado), sino la de lo interior y lo exterior. El Estado es la soberanía. Pero la soberanía sólo reina sobre aquello que es capaz de interiorizar, de apropiarse localmente". Si lo queer es inapropiable queda en esencia fuera de toda articulación hegemónica por parte del Estado y sus políticas serán siempre de hostilidad.

Pero no se trata de considerar irremediablemente imposible la hospitalidad, sino entender cómo poner en suspensión o volver inocuo su siempre presente componente de hostilidad (Berg, Fiddian-Qasmiyeh, 2018). Así, cabe preguntarse quién y en qué contexto uno puede convertirse en un anfitrión sea para cualquier migrante o para migrantes LGBT+. También es importante saber quién es visto como un extraño en función de consideraciones raciales, de clase, género o sexuales y analizar las relaciones entre los anfitriones y los huéspedes, para establecer su taxonomía y encontrar las formas que mejor acotan, suspenden o desactivan las tensiones y jerarquías que producen la hostilidad.

Las redes de hospitalidad pasan por lograr que las personas migrantes sientan que no están solas -como lo expresa Chantal en su testimonio-, por saber que hay un espacio de socialización al que se pertenece, en el proceso de tránsito y establecimiento definitivo. Muchas veces eso ocurre de manera más natural en una lógica de solidaridad entre migrantes o como lo establece el proyecto Refugee Hosts ${ }^{6}$ para el contexto de Siria, Palestina e Irak, entre refugiados (Fiddian-Qasmiyeh, 2016; Refugee Hosts, s/f). Para ello resulta útil la perspectiva de Jean Luc Nancy sobre "ser-con" y "ser-con-el otro" que desde la revisión del concepto de Mitsein de Heidegger busca construir una analítica coexistencial (Nancy, 2000; Nancy, Engelmann, 2019). Desde esta perspectiva, la conformación de caravanas y redes de hospitalidad son formas de construcción de lo común, es decir de una socialidad que excede -o que se manifiesta a pesar de- las categorías y definiciones del estado-nación (Tataryn, 2013).

Las caravanas son en sí ya una forma de hospitalidad entre migrantes, sea para proteger la seguridad del colectivo (autodefensa) o para mantener el ánimo y crear lazos de fraternidad, amor, respeto y unión. Además, como subconjuntos de la caravana, se crean grupos internos en las mismas conformados por integrantes LGBT+ para evitar o mitigar la discriminación por razones de orientación sexual e identidad de género; estos subconjuntos reflejan diferentes capas de hospitalidad entre migrantes. Otra de las formas

${ }_{6}$ El proyecto Refugee Hosts busca desde una perspectiva interdisciplinaria avanzar en la comprensión de los retos y oportunidades que se generan cuando las comunidades locales se involucran en actividades para mejorar la calidad de vida de poblaciones desplazadas debido a conflictos violentos en Medio Oriente. 
de hospitalidad asociadas a las caravanas es a partir de las organizaciones e iniciativas de la sociedad civil que construyen espacios de refugio, de los cuáles algunos son específicamente destinados a los miembros de la diversidad sexual e incluso de manera específica para personas trans.

\section{Conclusiones}

Las políticas migratorias de Estados Unidos y de México se han fortalecido y vinculado a través de la protección de la soberanía y la seguridad interna, lo cual ha impactado en la población migrante centroamericana indocumentada que transita, se refugia o asila en ambos territorios. Toda esta política está soportada en acciones y marcos normativos que incrementan la discriminación y la criminalización como un mecanismo para aumentar la hostilidad, la intolerancia y la xenofobia hacia de los migrantes centroamericanos en general.

Las redes de hospitalidad se pueden componer en perspectiva micro desde los ciudadanos de los países de tránsito, destino y retorno y los migrantes establecidos o nuevos. Además, existen diferentes capas de hospitalidad que se reducen cada vez en la medida que nos acercamos a lo alter y queer, por ello la capa de la caravana en su conjunto es una forma de hospitalidad entre inmigrantes, pero que excluye en cierta medida, si bien no del todo, la alteridad de los migrantes LGBT+ que deben construir su propia capa de hospitalidad frente a la hostilidad que prevalece hacia la diversidad sexual. Así, una mayor precariedad está asociada a una mayor otredad y a una menor posibilidad de formar parte de ciertas construcciones de la hospitalidad. El reconocimiento del otro es un determinante de las formas de hospitalidad y un mecanismo para desplazar o poner en suspenso en la medida de lo posible la hostilidad.

Por tanto, para construir redes viables de hospitalidad más allá de los márgenes de los estados-nación será fundamental comprender las motivaciones de las personas LGBT + para emprender un éxodo peligroso desde sus lugares de origen, las que son las mismas de todos los migrantes: la marginación, la violencia, la guerra, la falta de oportunidades, la discriminación por género y preferencias sexuales, entre otras; pero se añaden a estas otros procesos de subjetivación que se producen desde la decisión de migrar por parte de integrantes de colectivos LGBT + y sus experiencias en trayectos que han de recorrer. La necesidad de salir de un entorno patriarcal cis heteronormativo sexista y violento pasa por la necesidad de sobrevivir, pero también es un requisito para convertirse en lo que se desea, para devenir trans en un sentido amplio, volverse realmente mujer $u$ hombre y ser reconocidxs como tales $y$ construir una nueva subjetividad en un entorno con mejores condiciones para 
ello, inexistentes en sus lugares de origen. Estamos ante un derecho a devenir lo que se desea, afirmarse a sí mismxs y el deseo de existir.

Desde la perspectiva de la hospitalidad, solo el apoyo de las organizaciones de la sociedad civil resulta genuino, las acciones del gobierno mexicano en la deportación de inmigrantes centroamericanos son producto de la supeditación de la soberanía mexicana a la de Estados Unidos, una soberanía así subsumida a otra de carácter más general es no sólo incompatible con cualquier forma de hospitalidad, sino que deviene meramente en hostilidad. Aunado a ello, las muestras de rechazo a los migrantes LGBT+ por parte de miembros de la sociedad vuelven más difícil su situación al colocarles en una posición de vulnerabilidad doblemente mayor, se vuelven otrxs en su propia otredad. Las muestras de indiferencia, odio o rechazo que reciben no provienen únicamente de las autoridades y la sociedad por la que atraviesan, sino también de la propia caravana de la que pretenden formar parte para proteger su travesía. Han sido únicamente las organizaciones de la sociedad civil -tanto mexicanas como de Estados Unidos- las que han buscado tender los puentes entre los migrantes LGBT+ y los demás migrantes (Jiménez, 2019).

Dado que las soberanías de México y Estados Unidos se encuentran subordinadas a la coyuntura política del acontecimiento Trump, lo más probable será la permanencia de los migrantes en territorio mexicano de manera indefinida o permanente, ello es algo de lo que ya se percatan muchas organizaciones civiles que prevén los modos de dotar de refugio a los migrantes, tarea que en el caso de la comunidad migrante LGBT+ se vuelve aún más compleja. Las redes de apoyo y hospitalidad han surgido de manera espontánea recobrando ese imperativo ético frente al otro, pero probablemente no sean aún suficientes ni en extensión ni en el reconocimiento amplio de la otredad que se enmarca en las identidades LGBT+.

\section{Referencias bibliográficas}

AGUIRRE, Juan; JARAMILLO, Luis. El papel de la descripción en la investigación cualitativa. Cinta de moebio, n. 53, p. 175-189, 2015. Disponible en: < https:// dx.doi.org/10.4067/S0717-554X2015000200006>.

BIDA, Aleksandra. Derrida and "Hostipitality". In: BIDA, Aleksandra (ed.). Mapping Home in Contemporary Narratives. 2018, p. 119-131. Disponible en: <https:// doi.org/10.1007/978-3-319-97967-0_8>.

BERG, Mette Louise; FIDDIAN-QASMIYEH, Elena. Hospitality and hostility towards migrants: Global perspectives-An introduction. Migration and Society, v. 1, n. 1, p. 1-6, 2018.

BLEVINS, John. Hospitality is a queer thing. Journal of Pastoral Theology, v. 19, n. 2, p. 104-117, 2009. Disponible en: <https://doi.org/10.1179/jpt.2009.19.2.006>. 
BRANDARIZ, José; MENDIOLA, Ignacio. Introducción: una mirada interdisciplinaria a la frontera como espacio y proceso. Revista CIDOB d'Afers Internacionals, n. 122, p. 7-13, 2019. DOI: doi.org/10.24241/rcai.2019.122.2.7.

BUTLER, Judith. Precarious Life: The Power of Mourning and Violence. New York: Verso, 2004.

CANALES, Alejandro. Vidas Vulnerables. Migración, derechos humanos y políticas públicas en cinco zonas fronterizas de América Latina. México: Universidad de Guadalajara: Miguel Ángel Porrúa, Librero-editor, 2013.

CANALES, Alejandro; ROJAS, Martha. Panorama de la migración internacional en México y Centroamérica. Santiago de Chile: Naciones Unidas, 2018. Disponible en: <https://www.researchgate.net/publication/326008461_Panorama_de_la_ migracion_internacional_en_Mexico_y_Centroamerica $>$.

CANALES, Alejandro; FUENTES, Juan; DE LEÓN ESCRIBANO, Carmen. Desarrollo y Migración. Desafíos y oportunidades en los países del norte de Centroamérica. México: CEPAL, Naciones Unidas, 2019.

CARRASCO, Gonzalo. La migración centroamericana en su tránsito por México hacia Estados Unidos. Alegatos, n. 83, p. 169-194, 2013. Disponible en: < http:// kali.azc.uam.mx/alegatos/>.

CASILLAS, Rodolfo. Migraciones centroamericanas en México, semblanza de un proceso social emergente. Relaciones, v. 12, n. 46. p. 67-81, 1991. Disponible en: <https://www.colmich.edu.mx/relaciones25/files/revistas/046/ RodolfoCasillasR.pdf $>$.

CASTAÑEDA, Alejandra. ¿Qué es el Programa Frontera Sur? Observatorio de Legislación y Política Migratoria. Boletín 1. El Colegio de la Frontera Norte, Comisión de los Derechos Humanos México, 2016. Disponible en: <https:// observatoriocolef.org/infograficos/11464990-programa-frontera-sur-copy/> .

CASTAÑEDA, Xóchitl; OSORIO, Liliana; CASTAÑEDA, Nicté. El amor en los tiempos de las cóleras: respuestas humanitarias a las caravanas migrantes. Brújula Ciudadana, n. 105, p. 19-23, 2019. Disponible en: <https://www. iniciativaciudadana.org.mx/brujulaciudadana $>$.

CASEY, Edward S. The Ethics of the Face to Face Encounter: Schroeder, Levinas, and the Glance. The Pluralist, v. 1, n. 1, p. 74-97, 2006.

CENTRO POR LA JUSTICIA Y EL DERECHO INTERNACIONAL. Diagnóstico sobre los crímenes de odio motivados por la orientación sexual e identidad de género en Costa Rica, Honduras y Nicaragua. San José, C.R.: CEJIL, 2013. Disponible en: $<$ https://cejil.org/es/diagnostico-crimenes-odio-motivados-orientacion-sexuale-identidad-genero-costa-rica-honduras-y $>$.

COLECTIVO DE OBSERVACIÓN Y MONITOREO DE DERECHOS HUMANOS EN EL SURESTE MEXICANO. Informe del monitoreo de derechos humanos del éxodo centroamericano en el sureste mexicano: octubre 2018-febrero 2019. 2019. Disponible en: <https://vocesmesoamericanas.org/wp-content/ uploads/2019/05/InformeExodo_Final-web.pdf>. 
DELEUZE, Gilles. "Estado y máquina de guerra". Estafeta (blog), 2005. Disponible en: <http://estafeta-gabrielpulecio.blogspot.com/2012/02/gilles-deleuzeestado-y-maquina-de.html>. Acceso en: 09.11.2019

DELEUZE, Gilles; GUATTARI, Felix. Mil mesetas: Capitalismo y esquizofrenia. PreTextos, 1997.

DEL MONTE, Juan; MCKEE, Robert. Personas Migrantes en Tijuana frente al Covid-19: Impactos y consecuencias de las medidas sanitarias desde la perspectiva de los actores. Documentos de Contingencia 8. El Colegio de la Frontera Norte, 2020. Disponible en: <https://observatoriocolef.org/boletin/ personas-migrantes-en-tijuana-frente-al-covid-19-impactos-y-consecuenciasde-las-medidas-sanitarias-desde-la-perspectiva-de-los-actores/> .

DERRIDA, Jacques. Hostipitality. Angelaki, v. 5, n. 3, p. 3-18, 2000. DOI: https:// doi.org/10.1080/09697250020034706.

DERRIDA, Jacques; DUFOURMANTELLE, Anne. La hospitalidad. Argentina: Ediciones de la Flor, 2000.

EL COLEGIO DE LA FRONTERA NORTE. La caravana de migrantes centroamericanos en Tijuana 2018. Diagnóstico y propuestas de acción. Tijuana, Baja California: El Colegio de la Frontera Norte, 2018. Disponible en: <https://www.colef. $\mathrm{mx} /$ estudiosdeelcolef/la-caravana-de-migrantes-centroamericanos-en-tijuana2018-diagnostico-y-propuestas-de-accion/> .

FERNÁNDEZ DE LA REGUERA, Alethia. ¿Qué sucedió una vez que la primera caravana migrante salió de Chiapas? Violaciones a los derechos humanos durante los procesos de solicitud de refugio y detención migratoria en la frontera sur. In: FERNÁNDEZ DE LA REGUERA, Alethia; GANDINI, Luciana; GUTIÉRREZ, Eduardo; NARVÁEZ, Juan (coords.). Caravanas migrantes: las respuestas de México. México: Instituto de Investigaciones Jurídicas, Universidad Nacional Autónoma de México, 2019, p. 33-52.

FIDDIAN-QASMIYEH, Elena. Refugee-refugee relations in contexts of overlapping displacement. International Journal of Urban and Regional Research, 2016.

GIBSON, Sarah. Mobilizing hospitality: The ethics of social relations in a mobile world. New York: Routledge, 2016.

GÓMEZ, Amaral. Entre la espada y la pared: Movilidad forzada de personas salvadoreñas LGBT. Mediações - Revista de Ciências Sociais, v. XXII, n. 1, p. 130155, 2017. DOI: http://dx.doi.org/10.5433/2176-6665.2017v22n1p130.

HERRERA, Gioconda; SØRENSEN, Ninna. Migraciones internacionales en América Latina: miradas críticas a la producción de un campo de conocimientos. Presentación del dossier. Íconos. Revista de Ciencias Sociales, n. 58, p. 11-36, 2017. DOI: http://dx.doi.org/10.17141/iconos.58.2017.2695.

HERRERO, Montserrat. Políticas de la hospitalidad en el pensamiento de Jacques Derrida. Revista de Estudios Políticos, n. 180, p. 77-103, 2018. DOI: https://doi. org/10.18042/cepc/rep.180.03. 
IMMIGRATION ADVOCATES. National Immigration Legal Services Directory. (s.f). Disponible en: <https://www.immigrationadvocates.org/nonprofit/ legaldirectory/organization.565010-Santa_Fe_Dreamers_Project>. Acceso en: 07.08.2020.

INSTITUTO PARA LAS MUJERES EN LA MIGRACIÓN. Recursos para entender el Protocolo "Quédate en México". 2020. Disponible en: < https://imumi.org/wpcontent/uploads/2020/02/Qu\%C3\%A9date-en-M\%C3\%A9xico-2020-1.pdf>.

JIMÉNEZ, Mabel. "Queer in the Caravan: The Dangers LGBT Migrants Face in Search for Asylum". El Tecolote (blog), 07.06.2019. Disponible en: < http://eltecolote. org/content/en/features/queer-in-the-caravan-the-dangers-lgbt-migrants-facein-search-for-asylum/> . Acceso en: 15.11.2019

KELZ, Rosine. Political Theory and Migration. Concepts of non-sovereignty and solidarity. Movements. Journal for Critical Migration and Border Regime Studies, v. 1, n. 2, 2015. Disponible en: <http://movements-journal.org/issues/02. kaempfe/03.kelz--political-theory-migration-non-sovereignty-solidarity.html > .

KELZ, Rosine. The Non-Sovereign Self, Responsibility, and Otherness. Hannah Arendt, Judith Butler, and Stanley Cavell on Moral Philosophy and Political Agency. New York: Palgrave Macmillan, 2016.

La caravana migrante LGTB, discriminación dentro de la discriminación. The Objective, 26.11.2018. Disponible en: <https://theobjective.com/further/ caravana-migrante-lgtb-discriminacion > . Acceso en: 08.10.2020

LEVINAS, Emmanuel. Totalidad e infinito: Ensayo sobre la exterioridad. Salamanca: Sígueme, 2006.

LOVE, Alegra. Migrant Caravan: On the Other Side. 2019. Disponible en: < https:// www.esperanzaproject.com/2019/migration-americas/migrant-caravan-on-theother-side/>. Acceso en: 07.08.2020.

LUIBHÉID, Eithne. Queer/Migration: An Unruly Body of Scholarship. GLQ: A Journal of Lesbian and Gay Studies, v. 14, n. 2, p. 169-190, 2008.

MADURA, Lisa Marie. Global Migration and the Ethics of Hospitality. 2016. Disponible en: <https://scholarworks.unr.edu//handle/11714/2184>.

MARTíNEZ, Graciela; COBO, Salvador; NARVÁEZ, Juan. Trazando rutas de la migración de tránsito irregular o no documentada por México. Perfiles Latinoamericanos, v. 23, n. 45, p. 127-155, 2015. Disponible en: <http://www. scielo.org.mx/pdf/perlat/v23n45/v23n45a6.pdf >.

MASTROGIOVANNI, Federico. II. Migrantes y Zetas. In: MASTROGIOVANNI, Federico. Ni vivos, ni muertos: la desaparición forzada en México como estrategia de terror. México: Grijalbo, 2014, p. 57-75.

MECANISMO PERMANENTE DE MONITOREO DE LA FRONTERA SUR MÉXICOGUATEMALA. Boletín n. 2, 18.05.2018. Disponible en: < http://accssgt.org/ actividades/wp-content/uploads/2018/07/Boletin-Migracio\%CC\%81n-MTFMG2DA-2018.pdf $>$. 
MESA TRANSFRONTERIZA MIGRACIONES Y GÉNERO. ANÁLISIS Y VISIÓN 2018-2020. 2018. Disponible en: <https://transfronteriza.org/materiales/ publicaciones/>.

MICHAUD, Ginette. Derrida and Sexual Difference. In: RABATÉ, Jean-Michel. After Derrida: Literature, Theory and Criticism in the 21st Century, After Series. Cambridge: Cambridge University Press, 2018, p. 58-79. DOI: https://doi. org/10.1017/9781108539937.004.

MIROFF, Nick. Under coronavirus immigration measures, U.S. is expelling border-crossers to Mexico in an average of 96 minutes. The Washington Post, 30.03.2020. Disponible en: <https:/www.washingtonpost.com/immigration/ coronavirus-immigration-border-96-minutes/2020/03/30/13af805c-72c5-11eaae50-7148009252e3_story.html>. Acceso en: 09.07.2020.

MORALES, Abelardo. Centroamérica: los territorios de la migración y la exclusión en el nuevo siglo. Foreign Affairs Latinoamerica. Octubre, 2013. Disponible en: $\quad<$ http://revistafal.com/centroamerica-los-territorios-de-la-migracion-y-laexclusion-en-el-nuevo-siglo/> . Acceso en: 01.10.2013.

NANCY, Jean-Luc. Being singular plural. Stanford: Stanford University Press, 2000.

NANCY, Jean-Luc; ENGELMANN, Peter. Democracy and Community. John Wiley \& Sons, 2019.

NARVÁEZ, Juan. Ruta Transnacional: a San Salvador por los Ángeles. Espacios de interacción juvenil en un contexto migratorio. México: Universidad Autónoma de Zacatecas, Instituto Mexicano de la Juventud, Editorial Porrúa, 2007.

NARVÁEZ, Juan. Hasta topar con pared. Historia de la migración en tránsito por México. In: FERNÁNDEZ DE LA REGUERA, Alethia; GANDINI, Luciana; GUTIÉRREZ, Eduardo; NARVÁEZ, Juan (coords.). Caravanas migrantes: las respuestas de México. México: Instituto de Investigaciones Jurídicas, Universidad Nacional Autónoma de México, 2019, p. 12-54.

PENCHASZADEH, Ana. Hospitalidad y soberanía. Reflexiones políticas en torno de la filosofía de Jacques Derrida. Isegoría. n. 40, p. 177-190, 2009. DOI: https:// doi.org/10.3989/isegoria.2009.i40.653.

PORTMAN, Scott; WEYL, Daniel. Reasentamiento de refugiados LGBT en los Estados Unidos: Buenas prácticas emergentes. Migraciones forzadas, n. 42, p. 44-47, 2013. Disponible en: < https://www.fmreview.org/sites/fmr/files/FMRdownloads/ es/osig/RMF42completo.pdf>.

Rechazan a migrantes LGTB en Playas de Tijuana. La voz de la frontera, 11.11.2018. Disponible en: <https://www.lavozdelafrontera.com.mx/local/rechazan-amigrantes-lgtb-en-playas-de-tijuana-2652829.html>. Acceso en: 08.10.2020.

REFUGEE HOSTS. Refugee Hosts. Refugee Hosts, s.f. Disponible en: < https:// refugeehosts.org/>. Acceso en: 29.06. 2020.

SANTA FE DREAMERS. Santa Fe Dreamers Project. (s.f.). Disponible en: < http:// www.santafedreamersproject.org/what-we-do>. Acceso en: 07.08.2020. 
SERRANO, Arcelia. Entendamos a las caravanas migrantes. Los Ángeles Times, 03.12.2019. Disponible en: <https://www.latimes.com/espanol/politica/la-esopinion-entendamos-a-las-caravanas-migrantes-20190312-story.html>. Acceso en: 06.01.2020.

STILL, Judith. Derrida and hospitality: Theory and practice. Great Britain: Edinburgh University Press, 2013.

TATARYN, Anastasia. Revisiting Hospitality: Opening Doors beyond Derrida towards Nancy's Inoperativity. Law Text Culture, v. 17, p. 184-210, 2013.

VARGAS, Felipe de Jesús. El vía crucis del migrante: demandas y membresía. Trace, n. 73, p. 117-133, 2016. Disponible en: <http://www.scielo.org.mx/pdf/trace/ n73/2007-2392-trace-73-117.pdf>.

VALENZUELA, José Manuel. Caminos del éxodo humano: Las caravanas de migrantes centroamericanos. Ciudad de México: Gedisa, 2019.

VARELA, Amarela; MC LEAN, Lisa. Caravanas de migrantes en México: Nueva forma de autodefensa y transmigración. Revista CIDOB d'Afers Internacionals, n. 122, p. 163-185, 2019. Disponible en: <https://doi.org/10.24241/ rcai.2019.122.2.163>.

YEPEZ DEL CASTILLO, Isabel. Escenarios de la Migración Latinoamericana: la vida familiar transnacional entre Europa y América Latina. Papeles del CEIC, n. 2, p. 1-27, 2014. Disponible en: <https://www.redalyc.org/comocitar. oa?id $=76532158002>$. 